\title{
Editorial: Is the gut intrinsically abnormal in rheumatoid arthritis?
}

In an animal model of arthritis pigs fed a diet rich in fish protein increased their faecal count of Clostridium perfringens type $\mathrm{A}$ and developed joint disease which resembled rheumatoid arthritis by the presence of pannus, erosion of cartilage, and the formation of subcutaneous nodules. ' Increased antibody titres to $C$ perfringens antigen could be detected but these were not concomitant with the development of the exudative arthritis. As it has been shown that faecal counts of $C$ perfringens are increased in patients with rheumatoid arthritis, ${ }^{2}$ and that these counts fall during treatment with sulphasalazine, ${ }^{3}$ the suggestion is offered that the aetiology of rheumatoid arthritis may be found in the gut. Such a concept is not new but gains added attraction in the light of recent advances in our understanding of the role that klebsiella related antigens may play in the HLA-B27 associated arthropathies. Ten years ago it was proposed that the intestinal absorption of toxic antigens and other noxious substances produced by microbial processes in the gut might play a role in the aetiopathogenesis of rheumatoid arthritis'. ${ }^{+}$In addition, it has been suggested that in rheumatoid arthritis the gastrointestinal mucosa is "frail" and more susceptible than normal to the ulcerogenic effect of non-steroidal anti-inflammatory drugs." One may imagine that an abnormal gut mucosa could facilitate an immune response to antigenic material from the lumen. Hypotheses and imagination aside. what evidence is there that the gut is intrinsically abnormal in rheumatoid arthritis?

At a macroscopic level there is no convincing, evidence that the upper gastrointestinal tract is more frequently abnormal in patients with rheumatoid arthritis. Peptic ulcer disease, either definite (previous gastric surgery or verifiable radiological or endoscopic evidence) or probable (compatible clinical features and history of radiological or endoscopic diagnosis), has been shown to be no more common in patients with rheumatoid arthritis than in controls with osteoarthritis. ${ }^{7}$ The increased prevalence of peptic ulcer disease in both groups compared with the general population was considered to be an effect of drug treatment, particularly non-steroidal anti-inflammatory drugs. Unfortunately, macroscopic lesions correlated poorly with histological change, and although non-steroidal anti-inflammatory drugs are widely recognised to cause macroscopic damage, such as erosions and ulceration of the gastric mucosa, there are less data concerning their ability to cause microscopic damage. The data that are available are of questionable value. For example, one study, which suggested that flurbiprofen was not responsible for significant alterations in gastric mucosal histology, overlooked the fact that all the patients examined had abnormal light microscopy on entry into the study and that drugs taken immediately before the study were not stated, nor was mention made of a washout period. ${ }^{8}$

Histological change of the gastric mucosa is a common finding in patients with rheumatoid arthritis, but is non-specific. Chronic superficial gastritis was seen in $30 \%$ of biopsy specimens and chronic atrophic gastritis in $62.5 \%$, in a series of 59 unselected patients with rheumatoid arthritis." Chronic atrophic gastritis is, however, a common age related finding in the population. ${ }^{10}$ The ability of drugs to produce similar changes has not been well studied. Although reported to be more common in patients with Sjögren's syndrome, ${ }^{11}$ the glandular atrophy and lymphocytic infiltration of chronic atrophic gastritis resemble the histology of Sjögren's syndrome itself. The histology of chronic atrophic gastritis in those with Sjögren's syndrome was indistinguishable from that in patients with rheumatoid arthritis who did not have Sjögren's syndrome, leaving open the question of whether such changes were intrinsic to the Sjögren's syndrome.

Similar non-specific inflammatory changes have been demonstrated by light microscopy in colonic and rectal biopsy specimens." Examination of the rectal mucosa of patients with severe rheumatoid arthritis has shown ultrastructural changes such as proliferation of the rough endoplasmic reticulum, mitochondrial inclusions, association of the mitochondria with cytoplasmic filaments, and marked increase in the number of iron containing siderosomes. ${ }^{12}$ The significance of these findings is difficult to assess as all the patients studied had used suppository preparations of non-steroidal antiinflammatory drugs at some time, and these drugs are known to cause ultrastructural damage to cells, as shown in the gastric mucosa by apical membrane rupture, intracellular disorganisation, and cellular exfoliation. This ultrastructural damage occurs independently of any macroscopic or functional abnormality of the mucosa.

Fibreoptic colonoscopy allows access to and 
visualisation of the terminal ileum, which provides an opportunity to obtain histological material from that region. Ileocolonoscopy has been used to demonstrate histological inflammation in $82 \%$ of patients with reactive arthritis, ${ }^{13}$ but this was not shown in members of the control group, which included patients with rheumatoid arthritis. Both groups were taking non-steroidal anti-inflammatory drugs, thus excluding these agents as an important cause of this inflammation. This finding does not agree with studies which have shown that ${ }^{111}$ In labelled granulocytes accumulate in the terminal ileum and ascending colon of patients with rheumatoid arthritis. ${ }^{14}$ Accumulation of labelled granulocytes in this region has been interpreted as indicating a loss of ileal mucosal integrity due to the effect of non-steroidal anti-inflammatory drugs. Direct evidence of loss of mucosal integrity at a macroscopic or histological level is lacking, but it has been clearly demonstrated that these drugs do increase gut permeability to ${ }^{51} \mathrm{Cr}$ edetate. ${ }^{15}$ In control subjects the urinary excretion of an orally administered dose of ${ }^{51} \mathrm{Cr}$ edetate was raised within 48 hours of starting naproxen $1125 \mathrm{mg}$ daily; this raised excretion continued throughout the seven day treatment period and returned to normal 14 days after the drug was stopped. Might this increased permeability lead to increased antigen contact and so favour the persistence of arthritis? Probably not, as despite the widespread and increasing use of non-steroidal antiinflammatory drugs, the severity of rheumatoid arthritis is said to be declining.

It is not clear why only a small percentage of patients with rheumatoid arthritis develop peptic ulcer disease. It is tempting to speculate that a genetic predisposition may be of importance. Gastric mucus glycopeptide units have been shown to be smaller in patients with rheumatoid arthritis than in normal controls, ${ }^{16}$ though this may be an iatrogenic effect. Mucus glycoprotein composition may contribute to the upper gastrointestinal complications of treatment, however, as it is possible to predict which rats are susceptible to gastric ulceration when exposed to chronic aspirin administration. ${ }^{17}$ Rats with a lower percentage of high molecular weight mucus glycoprotein before the administration of aspirin $375 \mathrm{mg} / \mathrm{kg}$ daily had a significantly higher ulcer score than those with a high percentage of high molecular weight mucus glycoprotein when the stomach was examined after six months of oral aspirin administration. As the biochemical difference was present before drug administration a drug effect cannot be the explanation.

There is no evidence to date to suggest that the gut is intrinsically abnormal in rheumatoid arthritis, though it is clearly affected by the potent drugs employed in the treatment of the disease. The prevalence of peptic ulcer disease is no greater in $\overrightarrow{\overrightarrow{\vec{s}}}$ patients with rheumatoid arthritis than in those with osteoarthritis, indicating that the gut mucosa of patients with rheumatoid arthritis does not have an increased susceptibility to the effects of non-steroidab anti-inflammatory drugs. Whether such drugs cause microscopic damage remains an open question, and? further studies are required to assess the contribution $\overrightarrow{0}$ Sjögren's syndrome may make to the prevalence of chronic atrophic gastritis in patients with rheumatoid arthritis. Non-steroidal anti-inflammatory drugs have been shown to increase gut permeability, though the mechanism is unknown. A change in function, rather than of structure, appears the most likelyo explanation as ileocolonoscopy has failed to show either macroscopic or histological alteration of the terminal ileum. It is possible that variability in the quality of gut defence mechanisms may explain some of the gastrointestinal manifestations in rheumatoid arthritis. The hypothesis that the aetiology of rheumatoid arthritis lies in the intestine? must continue to remain a hypothesis until proved $\vec{\infty}_{\infty}^{\infty}$

Royal National Hospital

for Rheumatic Diseases, Upper Borough Walls, Bath BA1 1RL

\section{References}

1 Mansson I, Norberg R, Olhagen B, Bjorklund N E. Arthritis i pigs induced by dietary factors. Clin Exp Immunol 1971; 9? $677-93$.

2 Shinebaum R, Neumann V C, Cooke E M. Wright V. Comparison of faecal flora in patients with rheumatoid arthritis and controls. Br J Rheumatol 1987; 26: 329-33.

3 Neumann V C, Shinebaum R, Cooke E M, Wright V. Effects of sulphasalazine on faecal flora in patients with rheumatoif arthritis: a comparison with penicillamine. $\mathrm{Br} \mathrm{J}$ Rheumatos 1987; 26: 334-7.

4 Gullberg R. Possible role of alterations of the intestinal flora in rheumatoid arthritis. Rheumatology and Rehabilitation 1978; 1 t (suppl): 5-10.

5 Rainsford K. Side effects of anti-inflammatory/analgesic drugs은 epidemiology and gastrointestinal tract. Trends in Pharmaco logical Sciences 1984; 5: 205-8.

6 Rainsford K. An analysis of the gastrointestinal effects of non steroidal anti-inflammatory drugs, with particular reference to comparative studies in man and laboratory species. Rheuma tol Int 1982; 2: 1-10.

7 Malone D E, McCormick P A, Daly L, et al. Peptic ulcer in rheumatoid arthritis-intrinsic or related to drug therapy? $\operatorname{Br} \cong$ Rheumatol 1986; 25: 342-4.

8 Teixera A V, Pocas L, Serrao D. Study of the gastric mucosa in rheumatic patients before and after the administration of flurbiprofren. Br J Clin Pract 1981; 35: 222-7.

9 Marcolongo R, Bageli PF, Montagnami M. Gastrointestina? involvement in rheumatoid arthritis: a biopsy study. $J$ Rheuma@ tol 1979; 6: 426-40.

10 Strickland R G, Mackay I R. A reappraisal of the nature an $\mathbb{R}$ significance of chronic atrophic gastritis. Am J Dig Dis 1973; 18 426-40. 
11 Maury C P J, Tornroth T, Teppo A-M. Atrophic gastritis in Sjögren's syndrome. Morphological, biochemical and immunologic findings. Arthritis Rheum 1985; 28: 388-94.

12 Struthers G R, Hollywell C A. Morris C J, Walton K W, Bacon $P$ A. Ultrastructural changes in the rectal mucosa of patients with rheumatoid arthritis. Ann Rheum Dis 1985; 44: 625-30.

13 Cuvelier C, Barbatis C, Miclants H, de Vos M. Roels H. Veys E. Histopathology of intestinal inflammation related to reactive arthritis. Gut 1987; 28: 394-401.

14 Bjarnason I. So A, Levi A J, et al. Intestinal permeability and inflammation in rheumatoid arthritis: effects of non-steroidal anti-inflammatory drugs. Lancet 1984, ii: 1171-4.
15 Jenkins R T. Rooney P J. Jones D B. Bienenstock J. Goodacre $R \mathrm{~L}$. Increased intestinal permeability in patients with rheumatoid arthritis: a side effect of oral non-steroidal anti-inflammatory drug therapy. Br J Rheumatol 1987: 26: 103-7.

16 Hutton C W, Esplugues J. Dieppe P A. Clamp J R. Abnormal mucus glycopeptide in rheumatoid arthritis. $\mathrm{Br} J$ Rheumatol 1987: 26 (suppl 2): 30.

17 Bagshaw P F. Munster D J. Wilson J G. Molecular weight of gastric mucus glycoprotein is a determinant of the degree of subsequent aspirin induced chronic gastric ulceration in the rat. Gut 1987; 28: 287-93. 